\title{
L'arôme du Pont l'Evêque. Mise en évidence de constituants volatils quantitativement mineurs"
}

par

\author{
J.P. DUMONT, Christiane DEGAS et J. ADDA \\ Station Centrale de Recherches Laitières et de Technologie \\ des Produits Animaux, I.N.R.A. \\ 78350 Jouy-en-Josas (France)
}

\section{INTRODUCTION}

Dans un travail précédent consacré à l'étude des produits volatils neutres de différents types de fromages à pâte molle et à croûte lavée [1], nous avions eu l'occasion de présenter la liste des principaux constituants de l'arôme du Pont l'Evêque. Parmi les 32 composés cités, les plus significatifs semblaient devoir être le phénol, l'acétophénone et l'indole qui sont les produits quantitativement majeurs et certaines substances présentes en faibles quantités tels les esters du méthanethiol (acétate et propionate).

Une nouvelle étude récemment réalisée sur le Pont l'Evêque nous a permis d'allonger substantiellement la liste des composés mis en évidence et de confirmer l'importance des thioesters dans l'arôme de ce fromage.

\section{MATERIEL ET METHODES}

\section{Les fromages}

Le lot de Pont l'Evêque analysé était constitué de fromages fermiers fabriqués au lait cru. Les fromages, non écroûtés, ont été râpés à l'état congelé et soumis à une distillation sous vide selon les modalités précédemment décrites [2].

Les extraits aqueux contenus dans les pièges sont réunis et ramenés à un volume de 10 à $15 \mathrm{ml}$ par cryoconcentration. L'extrait concentré est alors amené à $\mathrm{pH}$ 9, saturé en $\mathrm{NaCl}$ et soumis à une série d'extractions par de petites quantités de Fréon 11.

\footnotetext{
* Ce travail a bénéficié du concours financier de la D.G.R.S.T. (contrat nº 7570574 ).
} 
L'excès de solvant est éliminé par distillation en utilisant une colonne de Vigreux. L'extrait, d'un volume de 200 à $300 \mu \mathrm{l}$, est alors partagé en deux parties, l'une étant concentrée et chromatographiée directement sur colonne capillaire $(150 \mathrm{~m}, \varnothing 0,75 \mathrm{~mm}$ d.i., SF 96 : Igepal Co $880,95: 5)$, l'autre étant soumise à un fractionnement sur gel de silice désactivé en utilisant les différents éluants dans l'ordre suivant :

- Fréon 11.

- Gradient d'éther éthylique dans le Fréon 11.

- Ether éthylique.

- Gradient de méthanol dans l'éther éthylique.

On recueille des fractions de $1 \mathrm{ml}$ dont on évalue pour chacune l'importance olfactive. Les fractions sont ensuite réunies par 2 ou 3 en fonction de leur caractène olfactif, concentrées et analysées en utilisant le couplage chromatographie en phase gazeuse - spectromètre de masse [3].

\section{RESULTATS}

On a pu mettre en évidence les 123 composés suivants (tab. 1).

L'étude olfactive des fractions séparées lors de la chromatographie sur gel de silice a permis de vérifier le rôle important des thioesters. En effet il est apparu que les fractions qui possédaient une odeur caractéristique de Pont l'Evêque étaient justement celles qui contenaient ces thioesters et, comme par ailleurs, ces composés sont élués de façon groupée sans autres contaminants que quelques esters oxygénés à 8 atomes de carbone et plus, on peut penser qu'ils sont responsables pour une bonne part de l'odeur caractéristique des fractions qui les contenaient.

\section{CONCLUSION}

Cette étude a permis de mettne en évidence un certain nombre de composants quantitativement mineurs de l'arôme qui passent généralement inaperçus. Elle fournit un exemple de l'intérêt des méthodes de fractionnement préliminaire des extraits d'arôme avant l'étude par couplage chromatographie-spectrométrie. L'intérêt du mode de fractionnement utilisé réside dans le fait que les composés sont séparés par familles chimiques et qu'il est très facile d'en évaluer la « qualité olfactive ".

Ces thioesters dont l'importance précédemment pressentie s'est trouvée confirmée font l'objet d'une étude plus approfondie de leurs caractéristiques organoleptiques qui sera publiée ultérieurement. 


\section{TABLEAU 1}

Alcools : Méthanol, propanol*, butanol*, 2-méthyl-1-propanol*, pentanol, 3-méthyl1-butanol*, hexanol, 2-phényléthanol* ; 2-butanol*, 2-pentanol*, 2-heptanol*, 2-nonanol*, 2-undecanol, 2-phényléthane-2-ol* ; isobornéol, $\alpha$-terpinéol.

Esters : Formate de butyle, d'isoamyle, de 2-butanol ; acétate d'éthyle*, de propyle, de butyle, d'isoamyle, de phényléthyle*, de 2-butanol ; propanoate d'éthyle*, de phényléthyle; butyrate d'éthyle, de propyle, d'isoamyle, de phényléthyle, de 2-butanol ; hexanoate d'éthyle*, de propyle, d'isoamyle, de 2-butanol ; octanoate de méthyle*, d'éthyle, d'isoamyle, de 2-butanol ; décanoate de méthyle, d'éthyle ; dodecanoate de méthyle.

Cétones : 2-butanone*, 2-pentanone, 2-hexanone, 2-heptanone*, 2-octanone, 2-nonanone*, 2-décanone, 2-undecanone, 2-dodecanone, 2-tridecanone; acétophénone*, 1-phényl-2-propanone ; 4-méthyl-3-penten-2-one ; 1-hydroxy-2-propanone.

Aldéhydes : Acétaldéhyde, benzaldéhyde ; 2-butenal, 2 pentenal.

Acétals: Diethoxyéthane, éthoxypropoxyéthane, acétal $M=132$, éthoxybutoxyéthane, éthoxypentoxyéthane, acétal $M=174$, éthoxyhexoéthane, acétal $M=188$, dibutoxyéthane, éthoxyphénoxyéthane.

Hydrocarbures : Hexane, méthyloctane, méthyldecane, undecane, pentaméthylheptane, dodecane, tridecane, tetradecane ; dodecène ; benzène*, toluène*, xylène*, éthylbenzène, méthyléthylbenzène, méthylisopropyl benzène ; naphtalène, méthylnaphtalène ; limonène, $\alpha$-pinène.

Composés soufrés : Méthanethiol acétate*, propanoate*, butyrate, isovalérate, hexanoate, octanoate, decanoate; disulfure de méthyle*, trisulfure de méthyle*; benzothiazole, méthylthiobenzothiazole; méthyléthylsulfonate, méthylméthanethiosulfonate; non identifié (m/e 43, 71, 41, 27, 118, 75).

Phénol et dérivés : Phénol*, crésol*, éthylphénol, propylphénol; phénylacétate, phénylbutyrate, phénylvalérate, non identifié $(M=164)$.

Composés chlorés : Chlorure de méthyle, chlorure de méthylène, chlorobenzène, dichlorobenzène, trichlorobenzène.

Hétérocycles : Indole*, méthylindole ; hexylfurane.

Ethers : Méthoxybenzène (anisole) ; méthoxypropenylbenzène (anéthole).

* Composés déjà cités dans [1].

D'ores et déjà, si l'on se rapporte aux seules données actuellement disponibles dans la littérature [5] qui attribuent respectivement au méthylthiohexanoate et au méthylthioheptanoate des seuils de perception dans l'eau de $3.10^{-4}$ et $2 \cdot 10^{-3}$ p.p.m., on peut penser que même à des concentrations très faibles, les thioesters ont un rôle important dans l'arôme du Pont l'Evêque. Leur mise en évidence et leur dosage posent donc un problème analytique délicat. Dans ces conditions, on peut s'expliquer pourquoi certains auteurs [4] qui étaient parvenus à mettre en évidence le rôle des composés soufrés dans les fromages à croûte lavée, n'aient pas soupçonné la présence des thioesters, leurs méthodes n'étant ni assez sensibles, ni assez spécifiques. 


\section{S u m m a r y}

Quantitatively minor volatile compounds were studied in Pont l'Evêque (a surface ripened cheese) using silica gel column fractionation prior to capillary GC Analysis. Among 123 compounds the homologous series of methyl thioesters seems to play a significant role in the aroma along with phenol, cresol and acetophenone previously reported.

Reçu pour publication en janvier 1976.

\section{Références}

[1] Dumont (J. P.), Roger (Sylviane), AdDA (J.) (1974). — Etude des composés volatils neutres présents dans les fromages à pâte molle et à croûte lavée. Le Lait, 54, 531-532, 31.

[2] Dumont (J. P.), AdDa (J.) (1972), - Isolement des constituants de l'arôme des fromages : comparaison de méthodes. Le Lait, 52, 515-516, 311.

[3] Dumont (J. P.), Roger (Sylviane), Cerf (Paule) Adda (J.) (1974). - Etude des composés neutres volatils présents dans le Camembert. Le Lait, 54, 538, 501.

[4] Grill (H.) Jr., Pattgn (S.), Cone (J. F.) (1966). - Aroma significance of sulfur compounds in surface ripened cheese. J. Dairy Sci., 49, 403.

[5] Guadagni (D. G.), Buttery (Ron G.), Harris (J.) (1966). - Odour intensities of hop oil components. J. Sci. Food Agr., 17, 143. 